Revue internationale P.M.E.

Économie et gestion de la petite et moyenne entreprise

Revue

internationale

PME

\title{
Le marketing est-il soluble dans la très petite entreprise ?
}

\section{Jean-Claude Pacitto et Pierre-André Julien}

Volume 19, numéro 3-4, 2006

URI : https://id.erudit.org/iderudit/1008502ar

DOI : https://doi.org/10.7202/1008502ar

Aller au sommaire du numéro

Éditeur(s)

Presses de l’Université du Québec

ISSN

0776-5436 (imprimé)

1918-9699 (numérique)

Découvrir la revue

Citer cet article

Pacitto, J.-C. \& Julien, P.-A. (2006). Le marketing est-il soluble dans la très petite entreprise ? Revue internationale P.M.E., 19(3-4), 77-110.

https://doi.org/10.7202/1008502ar

\section{Résumé de l'article}

Les toutes petites entreprises (TPE) ne manifestent à l'égard du marketing qu'un intérêt limité. Cette réticence ne doit pourtant pas être interprétée comme une résistance au changement ou la simple persistance d'un référent trop traditionnel en matière de gestion ; elle provient de comportements particuliers, notamment d'une relation particulière qu'entretiennent les TPE avec leurs clients et leur environnement. Cette relation se structure autour de la notion de proximité, proximité tant géographique que relationnelle et culturelle. Cette proximité aboutit à une relativisation d'un objectif important du marketing, soit la conquête de nouvelles clientèles.

À l'aide d'une enquête auprès de 376 TPE françaises, nous avons analysé les différentes raisons de cette réticence. Nous avons comparé cette enquête à d'autres études de même type. Les résultats confirment que les TPE dédaignent certaines pratiques de marketing sans toutefois délaisser d'autres formes de liens forts avec leurs clients. C'est en examinant ces liens que l'on sera le plus à même de proposer des solutions commerciales et de marketing adaptées à ces entreprises. 


\title{
Le marketing est-il soluble dans la très petite entreprise?
}

\author{
Jean-Claude PACITTO \\ Laboratoire IRG-Management \\ IUT Techniques de commercialisation, Université Paris XII
}

Pierre-André JULIEN

Institut de recherche sur les PME

Université du Québec à Trois-Rivières

MOTS CLÉS

Artisanat - Commercialisation - Marketing - Proximité - TPE

\section{LES AUTEURS}

JeAN-Claude Pacitto est maître de conférences à l'IUT techniques de commercialisation de Créteil, il s'intéresse à la gestion des très petites et des moyennes entreprises. Ses recherches portent sur l'innovation ainsi que sur le marketing dans ces structures. Plus globalement, ses travaux tentent de cerner les critères permettant de définir la PME selon des groupes particuliers (très petites entreprises, petites entreprises et moyennes entreprises). II effectue ses recherches au sein des laboratoires de l'IRG de l'Université Paris XII et de l'INRPME de l'Université de Trois-Rivières au Québec. Adresse: Laboratoire IRG-Management, IUT TC Université Paris XII, 61, avenue du Général-de-Gaulle, 94010 Créteil Cédex 1. Courriel: $<$ pacitto@cegetel.net>.

PIERRE-AndRÉ JUlien est professeur émérite à l'Université du Québec à Trois-Rivières, Institut de recherche sur les PME et titulaire de la Chaire Bell pour des PME de classe mondiale. II a publié à ce jour dans différentes langues 96 articles scientifiques dans des revues savantes et 23 ouvrages, notamment en économie des PME et en développement de l'entrepreneuriat dans les régions. II est expert auprès du Groupe de travail sur les PME de l'Organisation de coopération et de développement économiques (OCDE) à Paris. Adresse: INRPME, Université du Québec à Trois-Rivières, C.P. 500, Trois-Rivières, Québec, G9A 5H7. Courriel : <pierre-andre.julien@uqtr.ca>.

\section{RÉSUMÉ}

Les toutes petites entreprises (TPE) ne manifestent à l'égard du marketing qu'un intérêt limité. Cette réticence ne doit pourtant pas être interprétée comme une résistance au changement ou la simple persistance d'un référent trop traditionnel en matière de gestion; elle provient de comportements particuliers, notamment d'une relation particulière qu'entretiennent les TPE avec leurs clients et leur envi- 
ronnement. Cette relation se structure autour de la notion de proximité, proximité tant géographique que relationnelle et culturelle. Cette proximité aboutit à une relativisation d'un objectif important du marketing, soit la conquête de nouvelles clientèles.

À l'aide d'une enquête auprès de 376 TPE françaises, nous avons analysé les différentes raisons de cette réticence. Nous avons comparé cette enquête à d'autres études de même type. Les résultats confirment que les TPE dédaignent certaines pratiques de marketing sans toutefois délaisser d'autres formes de liens forts avec leurs clients. C'est en examinant ces liens que l'on sera le plus à même de proposer des solutions commerciales et de marketing adaptées à ces entreprises.

\begin{abstract}
Very small enterprises (VSEs) demonstrate limited interest in marketing. This should not, however, be interpreted as resistance to change or simply an overly traditional view of management. Although such a view is part of the relationship between VSEs and marketing, other factors appear to better explain observed behaviour, specifically the particular relationship VSEs have with their customers and their environment. This relationship is built around the concept of proximity, both geographical and cultural, which puts the pursuit of new customers - a primary marketing objective - in a different perspective.

We have analyzed the reasons for this lack of interest using a survey of 376 French VSEs. We compared this survey to other similar studies. The results confirm that VSEs reject certain marketing practices, but without neglecting other types of strong customer connections. It is by examining these connections that we can best propose business and marketing solutions to suit these enterprises.
\end{abstract}

\title{
RESUMEN
}

Las muy pequeñas empresas (MPE) manifiestan hacia el marketing un interes muy moderado. Esta reticencia no debe por lo tanto ser interpretada como una resistencia al cambio o la simple persistenciade un referente demasiado tradicional en materia de gestión; ello provienen de comportamientos particulares, especialmente de una relación particular que mantienen las MPE con sus clientes y su medio ambiente. Esta relación se estructura alrededor de la noción de proximidad, proximidad tanto geográfica como relacional y cultural.

Esta proximidad tiene éxito relativamente con un objetivo importante de la mercadotecnia, es decir la conquista de nuevos clientes. Con la ayuda de una investigación junto a 376 MPE francesas, nosotros analizamos los diferentes razones de esta resistencia. Nosotros comparamos esta investigación con otros estudios de el mismo tipo. Los resultados confirman que las MPE desprecian algunas practicas en la mercadotecnia, sin embargo dejan otras formas de fuerte vinculo con sus clientes. 


\section{ZUSAMMENFASSUNG}

Die Mikrounternehmen (TPE, très petite entreprise) zeigen gegenüber dem Marketing nur ein sehr geringes Interesse. Diese Reserviertheit darf aber nicht allgemein als Widerstand gegen Änderungen oder als Beharrlichkeit auf ein traditionelles Managementverständnis verstanden werden. Sie resultiert vielmehr aus einer Besonderheit des Mikrounternehmens, die in der Beziehung zu ihren Kunden und ihrer Umwelt besteht. Diese Beziehungen sind geprägt von geographischer und kultureller Nähe und relativieren eine wichtige Zielsetzung des Marketings, die Bedeutung der Akquisition neuer Kunden.

Mit einer Untersuchung bei 376 französischen Mikrounternehmen haben wir die verschiedenen Gründe der zurückhaltenden Einstellung gegenüber dem Marketing analysiert. Die Resultate haben wir dabei mit Ergebnissen ähnlicher Studien verglichen. Die Ergebnisse bestätigen, dass Mikrounternehmen verschiedene Praktiken des Marketings verschmähen, ohne jedoch andere Formen der Kundenpflege zu vernachlässigen. Erst durch die Analyse dieser starken Beziehungen können Vorschläge für ein angepasstes Marketing für Mikrounternehmen formuliert werden.

\section{Introduction}

Comme on le sait, la plupart des entreprises sont toutes petites. Plus de $70 \%$ d'entre elles ont moins de 10 employés et plus de $90 \%$, entre 0 et 49 employés, quel que soit le pays (OCDE, 2002,p. 7). Cela s'explique non seulement parce que très souvent le premier objectif de leur création est de procurer un emploi à l'entrepreneur et à sa famille et d'en conserver le contrôle pour cette même raison, mais aussi parce que leur localisation le plus souvent proche du lieu d'origine du ou des créateurs est liée au petit marché identifié auparavant. Que ce soit le cas d'une petite épicerie, d'un salon de coiffure, d'une imprimerie de faire-part et de cartes professionnelles, d'un petit atelier d'usinage pour quelques entreprises voisines, d'un abattoir pour les fermes du coin, par exemple, l'existence d'un marché déjà bien couvert ou une forte concurrence provenant de l'extérieur ne permettent pas à ces firmes d'espérer grandir, même si parfois les raisons de départ évoluent.

D'ailleurs, durant la phase du prédémarrage, cette localisation permet d'obtenir directement toutes sortes d'informations sur le marché potentiel tout en permettant de prendre rapidement contact avec les clients au moment et après la création pour compléter la mise en marché, au point que les techniques de marketing développées pour les grandes entreprises ne peuvent être utiles pour expliquer la création de ces très petites entreprises (TPE). D'autant plus que leur manque de ressources constitue un frein à l'adoption de telles techniques même si elles étaient dans certains cas nécessaires (Marchesnay, 1988; Carson et al., 1995).

Revue internationale P.M.E., vol. 19, $\mathrm{n}^{\text {os }}$ 3-4, 2006 
Cette faible propension des TPE à recourir aux techniques traditionnelles du marketing, si elle semble évidente à première vue, est-elle généralisée ? L'examen de la documentation consacrée à cette question pour les PME ne permet pas de répondre de manière catégorique à cette question. Cette documentation est toutefois peu développée, disparate dans ses applications (Werman, Span et Adams, 1989; Perreault, 2005; Romano et Ratnatunga, 1995; Marchini, 1997, 1998; Herrmann, 1998) et tend de surcroît à se concentrer sur les moyennes entreprises et à sous-estimer, sinon à négliger ainsi diverses formes de stratégies de mise en marché directes et originales et de veille commerciale par le réseautage (Gilmore, Carson et Grant, 2001).

Le constat doit donc être nuancé. Les PME et, plus particulièrement, les plus petites d'entre elles, ne sont pas forcément fermées à toute démarche marketing. Ce qui est en jeu ici, c'est une forme de marketing telle qu'elle a été théorisée à partir des années 1950 et 1960 et largement fondée sur la planification et les processus formels (Coviello, Brodie et Munro, 2000).

De ce point de vue, le fossé qui s'est constitué entre le développement du corpus scientifique et les pratiques réelles pose problème (Carson et Gilmore, 2001). De la même façon, la césure entre ce qui relève du marketing et ce qui relève du commercial tend à s'estomper, notamment par l'attention portée à l'approfondissement des liens avec la clientèle, et ce, dans les deux démarches. Il faut ajouter aussi que l'intérêt porté ces dernières années à l'entrepreneuriat, confondu trop souvent avec la création d'entreprise, n'a pas permis d'avancer dans la connaissance du marketing appliqué aux très petites entreprises.

L'attention privilégiée portée à la dynamique entrepreneuriale a certainement contribué à marginaliser l'étude de ces entreprises à croissance le plus souvent faible qui constituent pourtant une réalité incontournable des économies développées. Le caractère quelque peu déroutant de la gestion de ces entreprises n'a pas favorisé non plus la multiplication des études. De fait, et comme Hills (1987) l'a souligné, le peu d'importance de ces entreprises après leur création (sauf dans le cas des gazelles - Julien, Mustar et Estimé, 2001) dans la croissance de l'emploi ou de la production nationale devient vite facteur d'exclusion. Ajoutons que plus de $50 \%$ des TPE disparaissent avant cinq ans ${ }^{1}$ (Baldwin et Gorecki, 1991; OCDE, 2002).

À ce particularisme structurel et conjoncturel s'ajoutent, dans certains pays (notamment la France et l'Italie), des spécificités institutionnelles (le

1. Notons que ce chiffre a fait l'objet d'interprétations pour le moins contradictoires.

Revue internationale P.M.E., vol. 19, nos 3-4, 2006 
rattachement de certaines de ces entreprises au secteur de l'artisanat) qui ne sont pas sans influer sur la façon d'appréhender leur comportement (Auvolat, Lavigne et Mayère, 1985; Pacitto, Julien et Meier, 2002).

Toute réflexion sur le rapport qu'entretiennent les TPE avec le marketing débouche inéluctablement sur la question de leurs relations avec l'environnement en général et le marché en particulier (Théron, 2001). La réserve globale des dirigeants des TPE par rapport à une démarche marketing trouve son origine dans la perception même qu'ils ont du marché, laquelle découle largement des finalités que ces mêmes dirigeants assignent à leur organisation. Si les préoccupations économiques ne sont jamais absentes, elles sont étroitement imbriquées à d'autres valeurs qui le sont moins ou pas du tout, comme le désir d'accomplissement, l'indépendance, l'amour du travail bien fait (Gibb et Scott, 1986). La prédominance de ces finalités aboutit à ce que ces dirigeants ne se considèrent pas comme des acteurs évoluant dans des contextes strictement concurrentiels et qu'ils se sentent très peu concernés par des phénomènes comme la veille systématique stratégique et commerciale. Ainsi, au vocabulaire guerrier utilisé par les sciences de gestion, le dirigeant de la TPE préférera par exemple un vocabulaire plus pacifique où les concurrents deviennent des collègues ${ }^{2}$ (Marchesnay, 1988; Bentabet, Michun et Trouvé, 1999).

C'est l'objet de cet article d'essayer, à partir d'une enquête portant, entre autres, sur l'étude de la fonction commerciale et d'une analyse de la documentation, qu'elle soit de nature scientifique ou non ${ }^{3}$, de comprendre quels types de relations les TPE nouent avec leur environnement. Ou, plus précisément, cette étude s'intéresse aux facteurs, de nature structurelle ou culturelle, qui peuvent expliquer la réticence des dirigeants des TPE à adopter des démarches, sinon marketing, du moins commerciales.

En premier lieu, nous exposerons la méthodologie suivie. En deuxième lieu, nous présenterons les résultats touchant la fonction commerciale en distinguant six types de TPE. En troisième lieu, nous discuterons de ces résultats et les comparerons à d'autres études sur le même sujet en montrant comment un mode spécifique de gestion de l'environnement appelle à redéfinir, du point de vue de la TPE, la démarche marketing. La problématique en relation avec la documentation scientifique sera discutée systématiquement tout au cours de l'application, selon la démarche de la théorisation ancrée (Strauss et Corbin, 1998).

2. Surtout quand ces collègues évoluent dans de très petites structures.

3. En effet, beaucoup d'études dans ce champ d'expertise, auxquelles nous nous référerons plusieurs fois dans cette étude, ont été réalisées pour le compte d'organismes professionnels ou institutionnels. 


\section{Méthodologie}

L'étude ${ }^{4}$ présentée a été réalisée en France en 1997. Son objectif était d'appréhender les relations qu'entretiennent les très petites entreprises avec la fonction commerciale et de mesurer autant que possible l'impact de différentes dimensions sur cette fonction.

Nous avons tiré du répertoire SIRÈNE 376 petites entreprises et administré les questionnaires sur place. Parmi ces entreprises, 324 étaient localisées dans la région Île-de-France et 52 en Franche-Comté. Les enquêteurs ont interrogé soit l'entrepreneur (dans $95 \%$ des cas), soit le responsable commercial lorsqu'il y en avait (dans $5 \%$ des cas). Cent quarante-six entreprises avaient plus de 10 salariés, soit près de $40 \%$, donnant ainsi une taille moyenne de 14 salariés. La médiane s'établit à 9 et les deux quartiles à 6 et 17. L'échantillon se répartissait en trois activités : les imprimeurs (139 entreprises), les prothésistes (dentaires et autres: 121 entreprises) et les divers (ameublement, habillement, métallurgie: 116 entreprises).

On peut considérer l'échantillon comme représentatif quant aux tranches de taille, mais non sur le plan des métiers puisque les prothésistes sont surreprésentés, notamment par rapport aux imprimeurs (tableau 1). L'intervalle de confiance des résultats sur les proportions modales est de l'ordre de $5 \%$ en plus et en moins ${ }^{6}$. L'effectif s'est révélé suffisant et les phénomènes en jeu suffisamment typés pour permettre des analyses de variance hautement significatives. Les taux de réponse étaient d'autant meilleurs que la taille était importante. Il faut retenir que l'échantillon est constitué pour les deux tiers des entreprises des métiers, le tiers restant est issu de la population des 11 à 50 salariés.

4. L'enquête IRG a été réalisée dans le cadre des activités de recherche de l'Institut de recherche en gestion de l'Univeristé Paris XII.

5. Le répertoire SIRÈNE est en France un instrument administratif obligatoire où doivent être immatriculés toutes les entreprises et établissemnts soumis à des obligations fiscales et sociales.

6. C'est-à-dire qu'une valeur de $40 \%$ peut se lire «il y a $95 \%$ de chances pour que le bon chiffre se situe entre $35 \%$ et $45 \%$ ». 
TABLEAU 1

Structure de l'échantillon et de la population enquêtée

\begin{tabular}{|c|c|c|c|c|c|}
\hline 艾 & 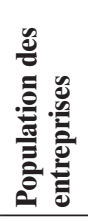 & 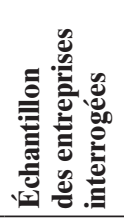 & 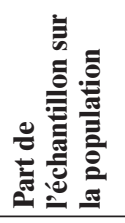 & 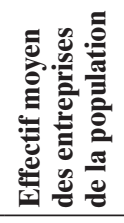 & 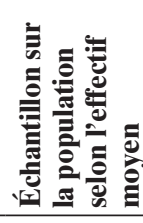 \\
\hline Divers & 525 & 116 & 22,1 & 16,5 & 0,92 \\
\hline Imprimeurs & 1001 & 139 & 13,9 & 15,2 & 0,97 \\
\hline Prothésistes & 271 & 121 & 44,6 & 12,8 & 0,83 \\
\hline
\end{tabular}

Les questions portaient sur l'attitude des dirigeants des TPE par rapport à la commercialisation à partir de diverses opinions sur lesquelles il leur était demandé de se prononcer sur une échelle, dans ce cas-ci inversée et purement ordinale, de 1 (tout à fait d'accord) à 5 (pas du tout d'accord), le chiffre médian 3 pouvant aussi bien s'interpréter comme de l'hésitation que de l'indifférence. Les questions d'opinions ont été construites à la suite d'un prétest provenant d'entretiens exploratoires menés avec 10 dirigeants de TPE et 3 responsables d'organisations professionnelles. Il a été aussi tenu compte des acquis de la documentation scientifique concernant le rapport qu'entretiennent les TPE avec la fonction commerciale et leur mode particulier de relation avec l'environnement. Dans cette perspective, le rapport au métier et le rapport à la clientèle ont été privilégiés mais toujours en relation avec d'autres problèmes comme la nécessité des outils commerciaux ou la conquête de nouveaux clients.

À partir des réponses apportées à ces questions, un des objectifs de l'enquête était de dégager des profils d'entreprises, profils permettant de mieux rendre compte de la diversité des TPE et de leur comportement. Ajoutons que compte tenu de la façon dont les questions ont été posées, on peut considérer qu'il y a un biais vers le 1; les répondants peu motivés par le sujet ont plus tendance à acquiescer qu'à s'opposer ou même à se placer en position médiane en optant pour une réponse de type neutre ou sans opinion.

Nous avons notamment confronté les résultats de cette enquête avec ceux de deux autres enquêtes parues récemment et réalisées en Italie et au Royaume-Uni (IMA, 2004; Fillis, 2002), enquêtes où les très petites entreprises constituaient l'essentiel des entreprises composant les échantillons. 
Dans la même perspective, nous avons utilisé les résultats les plus récents d'enquêtes menées en France auprès des TPE par des organismes professionnels ou d'études sur des thèmes proches de notre problématique. Cette comparaison nous permettra de mieux cerner le degré de contingence des résultats exposés et de nous interroger sur les «bonnes raisons » (Boudon, 1990) qui poussent les petites entreprises à peu investir le champ du marketing.

\section{RÉSULTATS}

\subsection{Les réponses aux questions}

Dans un premier temps, les variables sont examinées successivement et résumées au tableau 2, dans l'ordre du mieux accepté au plus souvent rejeté.

TABLEAU 2

Résultats sur les opinions portant sur les comportements commerciaux ou de marketing

\begin{tabular}{lcccc}
\hline Opinions & Divers & Imprimeurs & Prothésistes & Ensemble \\
\hline $\begin{array}{l}\text { Opinion 1: } \\
\text { «pas de dissociation des deux activités» }\end{array}$ & 1,47 & 1,43 & 1,56 & 1,48 \\
\hline $\begin{array}{l}\text { Opinion 2: } \\
\text { «offre de services personnalisés» }\end{array}$ & 1,73 & 1,72 & 1,91 & 1,78 \\
\hline $\begin{array}{l}\text { Opinion 3: } \\
\text { «avant tout la connaissance du métier» }\end{array}$ & 2,29 & 1,83 & 2,12 & 2,06 \\
\hline $\begin{array}{l}\text { Opinion 4: } \\
\text { «besoin de techniques modernes» }\end{array}$ & 2,34 & 2,23 & 2,64 & 2,39 \\
\hline $\begin{array}{l}\text { Opinion 5: } \\
\text { «pas les moyens d'avoir un responsable» }\end{array}$ & 2,90 & 2,78 & 3,00 & 2,89 \\
\hline
\end{tabular}

Cinq opinions portant sur des comportements commerciaux ou de marketing ont ainsi été proposées aux dirigeants.

Opinion 1. «Dans la très petite entreprise, on ne peut pas dissocier l'activité commerciale du reste. Pour établir un devis, l'acte commercial le plus important est de tout connaître de la production.» 
C'est la question la plus consensuelle (moyenne générale 1,48). On n'observe aucune esquisse de «courbe en $U$ » selon la taille ${ }^{7}$. Au total, toutefois, il y a un léger effet négatif sur la taille: plus l'entreprise est grande, plus elle est amenée à prendre quelque distance par rapport à cette affirmation qui ferait autrement l'unanimité.

Opinion 2. «L'avantage de la très petite entreprise est de fournir des services personnalisés face à une demande très diversifiée. C'est pourquoi les outils commerciaux de la grande entreprise ne sont pas adaptés, car ils ne sont pas fondés sur une relation personnelle avec le client.»

L'accord est général (moyenne 1,78). On note un petit effet négatif sur la taille et une légère défiance relative du secteur des prothèses. La courbe est globalement en «L»; il s'agit en fait d'un compromis entre le « $U$ » qui apparaît chez les producteurs de prothèses et la décroissance régulière et rapide des réponses lorsqu'on s'approche du «tout à fait d'accord» qu'on observe chez les imprimeurs.

Opinion 3. «Dans notre activité et surtout pour discuter avec le client, le plus important c'est de bien connaître le métier. Les techniques et les théories qu'on enseigne à l'université, cela vient bien après et on n'en a pas vraiment besoin.»

La tendance générale est favorable, mais la moyenne n'est que de 2,06 et donc légèrement en deçà du seuil correspondant au «plutôt d'accord». La proposition des «tout à fait d'accord» tombe en dessous des $50 \%$. La courbe est monotone et décroissante chez les imprimeurs, alors que le «U» est marqué dans les deux autres catégories. Cela finit par apparaître sur l'ensemble. L'effet taille est brouillé; seules les très petites entreprises et les imprimeurs se distinguent par un avis plus favorable que la moyenne.

Opinion 4. «Pour affronter la concurrence, la très petite entreprise doit grandir et trouver de nouveaux marchés. Pour cela, elle a besoin de techniques commerciales modernes et efficaces.»

Si l'on perçoit un accord d'ensemble, c'est du bout des lèvres (moyenne 2,39). Les réticences les plus fortes se rencontrent chez les plus

7. Une courbe en U montre d'abord beaucoup de réponses «très en accord» suivies de peu de réponses «un peu d'accord » et, à nouveau, beaucoup de réponses «pas du tout d'accord». Une telle vue de courbe permet de nuancer une moyenne globale qui ressemblerait à une autre courbe qui représenterait avant tout des réponses vers la moyenne, soit en cloche. De son côté, une courbe en L montre une proportion importante de répondants qui choisissent le «très en accord», alors que les autres se répartissent plus ou moins également sur les autres réponses. 
petites entreprises et chez les prothésistes, et ce, sans doute pour des raisons différentes. Il n'y a pas de courbe en «U» mais une décroissance lente, avec un point de départ peu élevé ( $35 \%$ de «très favorable») et une forte représentation du centre de la distribution.

Opinion 5. «Une très petite entreprise n'a pas les moyens d'avoir un responsable dont ce serait la seule activité; il vaut mieux utiliser une personne extérieure rémunérée à la commission.»

La moyenne est au milieu du spectre $(2,89)$ et on observe une parfaite courbe en «U», bien symétrique, peu d'avis médians et un regroupement sur les deux extrêmes.

\subsection{Une classification selon les opinions}

Les réponses sont déjà bien indicatrices de la façon qu'ont les TPE d'être critiques à l'égard des techniques marketing. Pour aller plus loin et pour mieux classifier ces opinions, nous avons utilisé l'algorithme du minimum de variance de Ward permettant la procédure de classification hiérarchique ascendante de groupes (cluster) de SAS.

Dans la normalisation a priori des variables entre 1 et 5, l'absence de queues de distribution trop fines prévient du risque de points isolés nuisibles à l'interprétation des classes. La taille de celles-ci et les possibilités d'interprétation priment les considérations de seuil de variance expliquée, permettant de fixer leur nombre à 6 . Cette technique permet ainsi de distinguer six profils de dirigeants résumés au tableau 3.

Commençons par le profil des défensifs, puisque c'est dans celui-ci que vont être regroupées les entreprises qui sont le plus proches de l'archétype artisanal (Candau, 1981). 


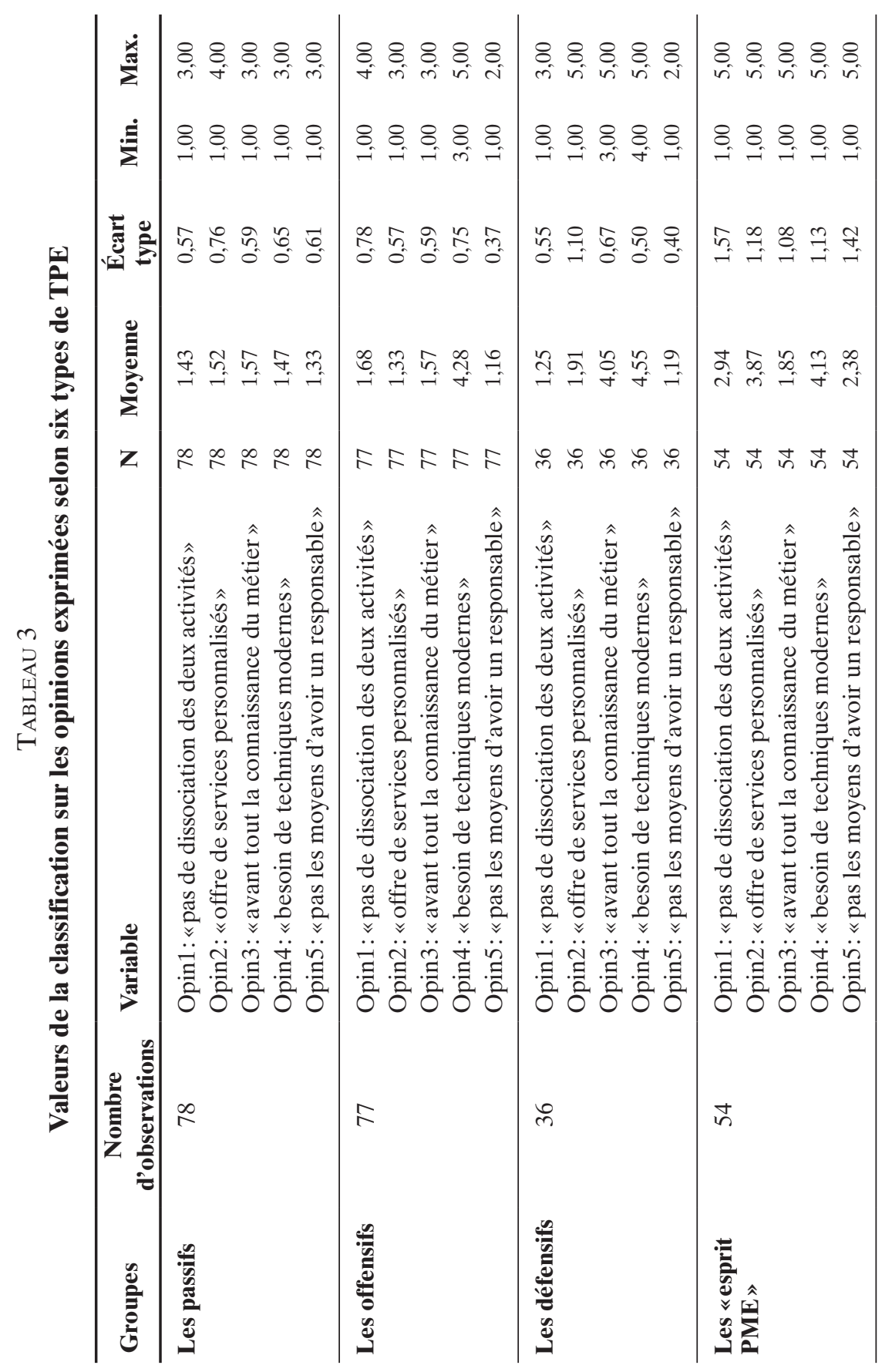

Revue internationale P.M.E., vol. 19, nºs 3-4, 2006 


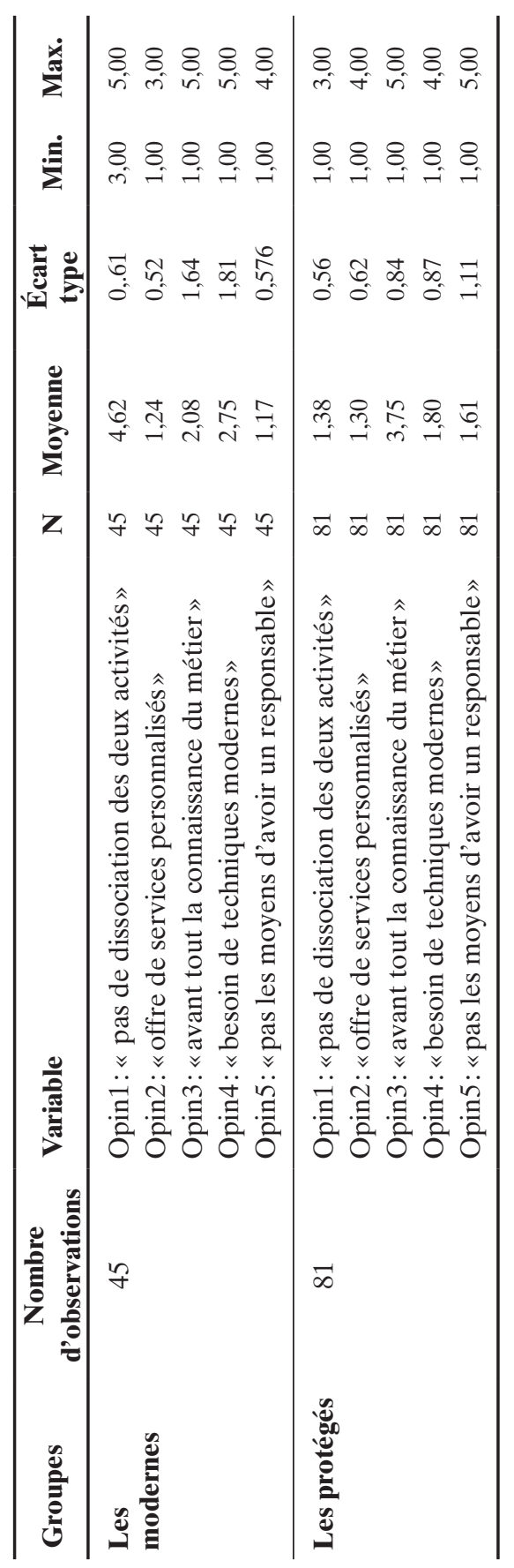

Revue internationale P.M.E., vol. 19, $\mathrm{n}^{\text {os }}$ 3-4, 2006

() 2006 - Presses de l'Université du Québec

Édifice Le Delta I, 2875, boul. Laurier, bureau 450, Québec, Québec G1V 2M2 • Tél.: (418) 657-4399 - www.puq.ca Tiré de: Revue internationale P.M.E., vol. 19, nos $3-4$, sous la direction de Louis Raymond • PME1903N Tous droits de reproduction, de traduction et d'adaptation réservés 
1. Les «défensifs» (36 entreprises): on est ici en présence d'entrepreneurs qui souhaitent conserver le contrôle sur toutes leurs activités (refus du commercial), qui refusent de voir leur entreprise croître et qui restent très attachés aux valeurs de la très petite entreprise (attachement au métier et à la personnalisation des prestations). Pour ces entrepreneurs, la dissociation activité commerciale/activité de production n'est pas envisageable.

2. Les «esprit PME» (54 entreprises): au contraire des précédents, les entrepreneurs de ce groupe ne sont pas fermés à la démarche commerciale, mais privilégient les solutions internes (un commercial salarié). Ils souhaitent grandir et rejettent les valeurs de la très petite entreprise, leur modèle restant celui de la moyenne et grande entreprise. La conquête de nouveaux clients et l'utilisation de quelques techniques commerciales modernes sont préférées à une personnalisation des liens avec les clientèles existantes.

3. Les «offensifs» (77 entreprises): ils partagent beaucoup de choses avec la catégorie précédente (envie de grandir, internalisation de la fonction commerciale) mais s'en détachent sur le problème des valeurs. En effet, ces entrepreneurs restent attachés à celles de la très petite entreprise, notamment pour tout ce qui touche à la relation à la clientèle et au lien qui est fait entre services personnalisés et avantage concurrentiel de la très petite entreprise.

4. Les «modernes» (45 entreprises) : ces entrepreneurs dissocient l'acte de production de celui de commercialisation. Ils sont à la recherche de nouvelles clientèles et sont, par là, favorables aux techniques qui leur permettront de les capter.

5. Les «protégés» (81 entreprises) : ils sont assez proches des entrepreneurs défensifs, mais se montrent moins réticents à la démarche commerciale (internalisation). Toutefois, ils ne dissocient pas (comme ces derniers) l'acte de production de l'acte commercial. Ils n'ont pas d'opinion tranchée quant à l'utilisation éventuelle de personnes extérieures rémunérées à la commission.

6. Les «passifs » (78 entreprises): ces entrepreneurs n'ont pas d'opinion marquée, mais restent néanmoins attachés aux valeurs de la TPE (attachement au métier et à la personnalisation des services). 


\section{Les facteurs d'indissolubilité ou le particularisme de la TPE}

Ces différents résultats doivent être toutefois expliqués.

\subsection{Le référent artisanal ${ }^{8}$}

Comme nous le soulignions dans l'introduction, la réticence, souvent constatée, des dirigeants des très petites entreprises à l'égard de la démarche commerciale réside dans le maintien, mais aussi le développement de valeurs qui paraissent souvent incompatibles avec les constituants d'une démarche entrepreneuriale classique. Peterson (1989) estime que les finalités (lieu d'accomplissement personnel et de plaisir, élément d'indépendance, etc.) qu'assignent ces dirigeants à leur entreprise ne favorisent pas l'adoption de démarches marketing ou, pour le moins, de démarches commerciales prononcées.

La non-utilisation du concept marketing trouve dès lors son origine dans le fait que le profit n'est pas le premier but de ces entrepreneurs. Comme l'avaient déjà fait remarquer entre autres Stanworth et Curran (1973), l'entrepreneur dominé par l'identité artisanale ne voit le profit que comme une conséquence de ses autres choix que sont la source de son emploi, l'indépendance, le choix des personnes avec lesquelles il travaille et la production de produits et services personnalisés dont il est fier (Le Cornu et al., 1996; Walter et Brown, 2004). Cozzi (1986) insiste aussi sur le style directionnel des dirigeants des petites entreprises qu'il estime peu compatible avec une orientation marketing. Ces conclusions rejoignent celles de Kinsey (1987) qui, dans une étude portant sur le marketing dans les petites entreprises industrielles écossaises, met en évidence le fait que, d'une part, les techniques de positionnement et de segmentation ne sont guère utilisées et que, d'autre part, les dirigeants de ces entreprises ne considèrent pas l'absence de démarche marketing comme une faiblesse.

D'un point de vue institutionnel, il est évident que le rattachement d'une grande part des TPE au secteur de l'artisanat n'est pas sans conséquence sur le développement de cultures d'entreprises particularistes ${ }^{9}$. Ce secteur

8. Le terme artisanal ne renvoie pas ici à la seule réalité institutionnelle, variable selon les pays, mais plutôt à son acceptation culturelle, c'est-à-dire à une manière particulière d'envisager l'activité structurée autour d'une relation privilégiée au métier (Fillis, 2002).

9. Le particularisme artisanal consiste à développer une conception autre de l'entreprise et notamment de la grande entreprise en affirmant un rapport privilégié au travail et au client. 
s'est, en effet, structuré autour du rejet d'une forme particulière d'économie de marché et d'une méfiance à l'égard des phénomènes de concurrence et d'adaptation obligée (David, 1998; Zdatny, 1998).

L'artisan préfère mettre l'accent sur des valeurs particulières plutôt que sur les seuls arguments économiques (la recherche de la croissance à tout prix, par exemple). Cela n'a d'ailleurs pas été sans influer sur le type d'études consacrées aux entreprises de ce secteur. Ainsi, si l'on trouve nombre de monographies mettant en relief la spécificité culturelle de l'artisanat, on ne rencontre, en revanche, que peu d'études qualitatives ou quantitatives qui traitent des problèmes économiques ou de gestion. On peut penser que la crainte d'une normalisation avec les autres entreprises et la fin, de ce fait, des spécificités structurelles de l'artisanat, ont largement contribué à façonner un état d'esprit où les préoccupations économiques n'ont pas toujours dominé. Toutefois, ce référent artisanal se rencontre aussi chez les entreprises n'appartenant pas au secteur des métiers.

La persistance au sein de la population des TPE d'un archétype artisanal (Candau, 1981), caractérisé par la focalisation du dirigeant sur l'aspect avant tout technique (le métier), n'est pas sans poser problème lorsque ces entreprises doivent relever un certain nombre de défis, dont celui de la croissance. Ainsi, dans une autre enquête portant sur 78 petites entreprises écossaises, Rosa et Hale (1990) ont pu montrer comment ce qu'ils nomment l'idéologie artisanale agit comme une barrière au développement des activités.

Dans son étude portant sur les barrières à l'internationalisation des entreprises artisanales britanniques, Fillis (2002) montre qu'au-delà du manque de ressources c'est bien ce référent artisanal qui est en jeu. C'est le rapport particulier que les propriétaires-dirigeants entretiennent avec leur métier qui constitue le principal obstacle à l'internationalisation, à l'adoption de démarches marketing proprement dites. Avant d'être un produit à mettre sur le marché, toute réalisation artisanale est d'abord une création unique et les impératifs de la commercialisation ne doivent pas altérer les paramètres de cette création. Les techniques de commercialisation ne sont en général acceptées que si elles ne remettent pas en cause ce rapport particulier; elles ne sont utilisées que si elles favorisent l'insertion du produit dans son environnement et non si elles président à sa conception même.

Les résultats révélés par l'enquête confirment ces développements. On y voit bien que la grande majorité des TPE enquêtées souscrivent à l'opinion (cf.2.1) selon laquelle «dans la très petite entreprise, on ne peut pas dissocier l'activité commerciale du reste $[. .$.$] ». La maîtrise du processus productif$ 
constitue la priorité des dirigeants de ces entreprises. On note ici un effet de taille significatif: plus les entreprises sont petites, plus elles souscrivent à cette opinion.

De la même façon, les plus petites entreprises de l'échantillon souscrivent majoritairement à l'opinion selon laquelle «dans l'activité et surtout pour discuter avec le client le plus important c'est de bien connaître le métier $[. .]$.$» . Les techniques liées à la commercialisation en général sont$ considérées comme secondaires, du moins par les plus petites entreprises de l'échantillon.

D'un point de vue général, on se rend compte que la vision de l'entrepreneur est dominée par le référent technique: créer son entreprise ou développer son affaire, c'est d'abord maîtriser une activité et plus globalement un métier.

Une enquête française portant sur 1016 TPE industrielles mettait aussi en lumière la persistance de ce référent chez les dirigeants des TPE industrielles (Kaminski, 1995). Ce que les dirigeants des TPE valorisent avant tout, c'est leur savoir-faire, et les valeurs auxquelles ils déclarent adhérer sans hésitation sont celles qui les attachent à leur métier. Le désir de croître reste une option très minoritaire.

En bref, la plupart des TPE pérennes ${ }^{10}$ restent des entreprises stables, comme l'avait bien montré Davidsson (1989) et au sens de ce qu'entend Marchini (1998). Pour elle, la volonté de ne pas croître entraîne inéluctablement des conséquences sur les modes de gestion, ce qui distingue ces entreprises de celles émergentes ${ }^{11}$ qui, par leurs choix stratégiques, privilégient la croissance et des modes de gestion en cohérence avec ces choix. On peut penser que la méfiance à l'égard des démarches commerciales en général est directement liée à ce souhait: le développement d'une capacité commerciale autonome étant associé à la croissance de l'entreprise.

Dans une perspective analogue, la réserve des dirigeants de la TPE envers l'engagement concurrentiel tient aussi au fait qu'ils en perçoivent négativement les répercussions au sein de la structure de leur entreprise. On peut constater en effet que les réponses apportées à l'opinion selon laquelle «Pour affronter la concurrence, la très petite entreprise doit grandir et trouver de nouveaux marchés. Pour cela, elle a besoin de techniques commerciales modernes et efficaces » varient avec la taille de l'entreprise, les dirigeants des plus petites entreprises étant extrêmement réservés au sujet de cette affirmation.

10. Soit celles qui passent à travers les premières années.

11. Celles qui veulent croître. 
Il est donc vain de corréler absence de démarche marketing et manque de dynamisme de l'entreprise. Ce qui est recherché par le dirigeant de la TPE stable, c'est un certain état d'équilibre, équilibre qui passe par le contrôle de son organisation ainsi que par la maîtrise de son environnement.

\subsection{Le rapport au marché : de la méprise au particularisme}

\subsection{La méprise}

Une démarche marketing nécessite deux conditions: la première, c'est la compréhension de la concurrence. Même si cette connaissance est souvent subjective, le développement d'une stratégie concurrentielle passe toujours par cette étape (Ohmae, 1983; Narver et Slater, 1990). La seconde, c'est la volonté de l'entreprise de bien délimiter sa demande, son marché et de moduler son offre par rapport à ceux-ci (Kotler, 1999). Or, dans le cas de la TPE, ces deux conditions sont rarement réunies. Comme il a été dit précédemment, la notion même de concurrence est le plus souvent ignorée et l'identification de la demande, rarement réalisée.

C'est pourquoi la notion de marché apparaît comme étrangère à leur culture. Comme l'écrit Letowski (1987, p. 132): «Nous avons fréquemment utilisé le terme de marché. En réalité, celui de clientèle serait plus juste. Le terme marché demeure un terme très abstrait pour la plupart des artisans. Il évoque plutôt celui de marché forain, là où sont rassemblés la plupart des artisans ou commerçants dans un lieu donné.»

Pourtant, il nous a tout de même paru intéressant de garder dans le contenu de notre enquête un questionnement ayant trait au marché sans chercher à adapter ce terme aux réalités de la TPE. C'était là la meilleure façon de percevoir ce que précisément ces entreprises entendaient par marché.

Le tableau 4 ajoute aux distinctions relatives à la taille celles touchant aux profils (cf. 2.2). On peut y voir que la quasi-totalité des entreprises de notre échantillon déclarent connaître leur marché, alors que $80 \%$ d'entre elles estiment ne pas avoir besoin d'études de marché. Ces pourcentages ne varient pas beaucoup, quels que soient les métiers, la taille ou les profils. Ces chiffres ne doivent pas faire illusion. Ils laissent à penser que sont, de fait, confondues deux réalités, le client et le marché (Marchesnay, 1988; Pacitto et Tordjman, 2000; Théron, 2001), et que, au contraire des moyennes et grandes entreprises, il n'est pas synonyme d'espace à conquérir. En répondant massivement et positivement à cette question, les entreprises montrent qu'au fond celle-ci n'a probablement pas de sens pour elles. 
Il faut noter que l'on retrouve des résultats à peu près similaires dans l'étude menée par l'Istituto marketing agroalimantare (IMA, 2004) portant sur 101 PME italiennes et dont plus de la moitié avait un effectif inférieur à 20 salariés $^{12}$. Dans cette enquête, $70 \%$ des entreprises déclarent ne pas utiliser de données relatives au marché ni même les susciter. Lorsqu'on interroge ces entreprises sur les services jugés prioritaires, la recherche d'information sur les marchés et les secteurs n'arrive qu'en quatrième position (sur sept). On remarquera que les services promus sont ceux qui sont perçus comme concrets (aide à la promotion, à l'internationalisation) et reliés à des opérations précises et ponctuelles.

TABLEAU 4

Connaissance du marché par les dirigeants des TPE

\begin{tabular}{|c|c|c|c|c|c|c|c|c|c|}
\hline & \multicolumn{4}{|c|}{ Un marché bien connu? } & \multicolumn{5}{|c|}{$\begin{array}{l}\text { Le besoin } \\
\text { d'études de marché? }\end{array}$} \\
\hline & \multicolumn{2}{|c|}{ NON } & \multicolumn{2}{|l|}{ OUI } & \multicolumn{2}{|c|}{ NON } & \multicolumn{2}{|c|}{ OUI } & \multirow{2}{*}{$\begin{array}{l}\text { TOTAL } \\
\mathbf{N}\end{array}$} \\
\hline & $\mathbf{N}$ & $\%$ & $\mathbf{N}$ & $\%$ & $\mathbf{N}$ & $\%$ & $\mathbf{N}$ & $\%$ & \\
\hline TOTAL & 25 & 6,6 & 351 & 93,4 & 304 & 80,9 & 72 & 19,1 & 376 \\
\hline \multicolumn{10}{|l|}{ Taille } \\
\hline 1 à 6 & 9 & 9,3 & 88 & 90,7 & 84 & 86,6 & 13 & 13,4 & 97 \\
\hline 7 à 10 & 11 & 8,3 & 122 & 91,7 & 102 & 76,7 & 31 & 23,3 & 133 \\
\hline 11 à 20 & 3 & 3,8 & 77 & 96,3 & 67 & 83,8 & 13 & 16,3 & 80 \\
\hline Plus de 20 & 2 & 3,0 & 64 & 97,0 & 51 & 77,3 & 15 & 22,7 & 66 \\
\hline Opinions & 6 & 7,6 & 73 & 92,4 & 64 & 81 & 15 & 19,0 & 79 \\
\hline Offensifs & 1 & 1,3 & 77 & 98,7 & 56 & 71,8 & 22 & 28,2 & 78 \\
\hline Défensifs & 4 & 11,1 & 32 & 88,9 & 32 & 88,9 & 4 & 11,1 & 36 \\
\hline $\begin{array}{l}\text { Esprit } \\
\text { PME }\end{array}$ & 3 & 5,4 & 53 & 94,6 & 44 & 78,6 & 12 & 21,4 & 56 \\
\hline Modernes & 4 & 8,9 & 41 & 91,1 & 43 & 95,6 & 2 & 4,4 & 45 \\
\hline Protégés & 7 & 8,5 & 75 & 91,5 & 65 & 79,3 & 17 & 20,7 & 82 \\
\hline
\end{tabular}

12. L'effectif moyen des entreprises de l'échantillon était de 30 salariés avec un chiffre d'affaires inférieur à 10 millions d'euros.

Revue internationale P.M.E., vol. 19, nos 3-4, 2006 
Pour autant, il serait prématuré de conclure à un rejet global des démarches informationnelles. C'est ici un certain type de démarche (formelle et rationnelle) qui est en cause.

D'autres études montrent, par ailleurs, que des démarches informationnelles existent, mais qu'elles se développent dans un cadre très informel (Scase et Goffee, 1980; Smeltzer, Fann et Nikolaisen, 1988; Julien, 1995). L'évaluation relève le plus souvent d'un processus intuitif et très subjectif basé, d'une part, sur l'expérience de l'entrepreneur et sur ses propres sensations (Dennis, 1987) et, d'autre part, sur la proximité particulière avec les clients. Cette démarche informationnelle va être grandement complétée en mobilisant des réseaux personnels du dirigeant (Gilmore, Carson et Grant, 2001; Julien, Lachance et Morin, 2005).

Si ces phénomènes concernant la veille commerciale sont de plus en plus étudiés, ils nécessitent la mise en œuvre de méthodologies spécifiques pour la TPE peu compatibles avec des démarches strictement quantitatives. Ainsi, Filion (1996), dans son étude sur les travailleurs autonomes du Québec, montre bien comment l'étude des réseaux de ces travailleurs permet de mettre en lumière de véritables systèmes marketing et que le différentiel de compétitivité constaté entre ces travailleurs résulte de leur capacité ou non à susciter ou à s'insérer dans ces réseaux. D'autres auteurs (O'Donnell et al., 2002) arrivent aux mêmes conclusions.

De la même façon, l'étude de la concurrence n'est pas jugée utile, celle-ci étant souvent perçue comme située dans un ailleurs difficilement localisable. Seuls les concurrents locaux sont généralement connus, mais à la seule condition qu'ils fabriquent des produits étroitement similaires à ceux de l'entreprise (Chicha et Julien, 1980). On remarquera aussi que le profil de l'entrepreneur ne modifie pas en profondeur la réponse apportée à la nécessité ou non d'études de marché.

Une étude française récente (Nexima, 2005) portant sur le marketing dans les PME confirme ces résultats. La plupart des entreprises interrogées (dans une majorité de TPE industrielles) se déclarent peu intéressées par l'étude de la concurrence en particulier et de tout ce qui a trait à l'environnement en général. Dans l'étude italienne précitée (IMA, 2004), près de $45 \%$ des entreprises déclarent ne pas effectuer de veille concurrentielle systématique; ce résultat est d'autant plus étonnant que ces entreprises évoluent dans le secteur agroalimentaire où la concurrence est une réalité pour la plupart d'entre elles. L'attention est portée sur la clientèle et sur les concurrents locaux, mais va rarement au-delà. 


\subsubsection{Méprise ou réalisme?}

La non-orientation marché des TPE tient pour beaucoup à leur mode d'insertion dans l'environnement et à la nature du processus de création des ressources. Ce dernier est, pour la TPE, étroitement dépendant des demandes qui lui sont adressées. La TPE raisonne en fonction de cette demande effective et non pas en fonction d'un éventuel positionnement du produit par rapport à des demandes plus indifférenciées ou potentielles, ou encore à des offres concurrentes. De toute façon, ce processus de création de ressources est tellement orienté et, par là même, dépendant, que son adaptation à d'autres demandes nécessiterait un effort d'investissement que très peu de TPE seraient susceptibles d'assumer. Enfin, on ne doit pas sous-estimer l'un des problèmes fondamentaux des dirigeants de TPE, celui de la gestion du temps liée à la pluralité des rôles assumés par eux (Chicha et Julien, 1980; Candau, 1981) $)^{13}$. Tout ceci explique, en dernier lieu, l'origine des phénomènes de dépendance à la clientèle, comme on le verra plus loin.

Lorsqu'on examine le rapport des TPE au marché, une autre difficulté surgit, soit celle du savoir-faire (Siméoni, 1998). Celui-ci est envisagé plus comme un tour de main, une référence identitaire (Letowski, 1987) que comme une compétence fondamentale, un cœur de métier (Deret, 1999) exploitable dans d'autres applications. Souvent, ce savoir-faire se confond avec le produit, ce qui rend encore plus difficile la dissociation des deux.

En dernier lieu, ce qui fait le plus défaut à la TPE, ce n'est pas ce fonds de compétence centrale (Amendola et Gaffard,1988), mais la conscience d'en posséder un et que celui-ci pourrait déboucher sur d'autres applications, ne serait-ce que dans le cadre de la relation privilégiée avec la clientèle. Toute démarche commerciale devra nécessairement passer par cette étape préalable: définir ce que l'on peut faire (de plus) et, à partir de là, examiner les éventuels nouveaux débouchés. Pour l'entrepreneur, dans ce cas, le savoirfaire ne se confondra pas avec le produit; il constituera une compétence qui peut être déployée ailleurs. En outre, le produit devra pouvoir faire l'objet, de la part du client, d'une évaluation. La notion de satisfaction devra être pensée réciproquement, dans une relation, et non plus seulement comme la rencontre unilatérale entre un savoir-faire et un besoin donné. Il faudra s'appliquer à définir au mieux les compétences à l'œuvre et envisager ainsi les types d'application qui peuvent en résulter et, par là même, les clientèles qui pourront être servies.

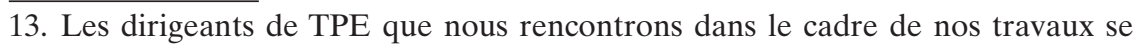
plaignent systématiquement du manque de temps.

Revue internationale P.M.E., vol. 19, $\mathrm{n}^{\text {os }}$ 3-4, 2006 
C'est seulement lorsque cette étape sera franchie que l'on pourra envisager d'examiner les demandes spécifiques du marché. De ce point de vue, on peut penser que la communication commerciale de la TPE devrait davantage porter sur la ou les compétences centrales de l'entreprise; d'autant plus pour celle en position de sous-traitance, comme dans une bonne part de la population étudiée dans le cadre de cette enquête, qui n'a pas ou qui a peu de contacts avec le consommateur final.

\subsubsection{La conséquence : le commercial aux mains du dirigeant}

Les développements précédents révèlent l'extrême réticence des dirigeants des TPE à dissocier la commercialisation de la production ainsi que la difficulté pratique à intermédiariser la relation entre les clients et l'entreprise. En conséquence, de façon logique et structurelle dans le cas des TPE, la fonction commerciale est très majoritairement gérée par le dirigeant, comme on peut le voir au tableau 5.

TABleau 5

Qui s'occupe de la fonction commerciale dans les TPE?

\begin{tabular}{|c|c|c|c|c|c|c|c|c|c|}
\hline & \multicolumn{2}{|c|}{ 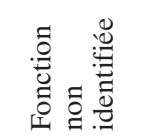 } & \multicolumn{2}{|c|}{ 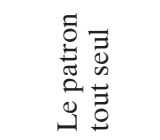 } & \multicolumn{2}{|c|}{ 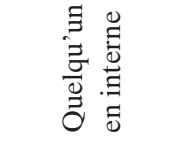 } & \multicolumn{2}{|c|}{ 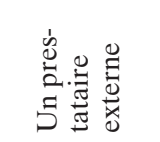 } & \multirow{2}{*}{ 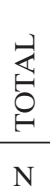 } \\
\hline & $\mathrm{N}$ & $\%$ & $\mathrm{~N}$ & $\%$ & $\mathrm{~N}$ & $\%$ & $\mathrm{~N}$ & $\%$ & \\
\hline \multicolumn{10}{|l|}{ Taille } \\
\hline 1 à 6 & 24 & 24,7 & 55 & 56,7 & 14 & 14,4 & 4 & 4,1 & 97 \\
\hline 7 à 10 & 30 & 22,6 & 63 & 47,4 & 34 & 25,6 & 6 & 4,5 & 133 \\
\hline 11 à 20 & 15 & 18,8 & 27 & 33,8 & 36 & 45,0 & 2 & 2,5 & 80 \\
\hline Plus de 20 & 5 & 7,6 & 18 & 27,3 & 33 & 50,0 & 10 & 15,2 & 66 \\
\hline Total & 74 & 19,7 & 163 & 43,4 & 117 & 31,1 & 22 & 5,9 & 376 \\
\hline
\end{tabular}

Pour plus de $80 \%$ des entreprises de moins de six salariés, c'est soit l'entrepreneur seul qui se charge de la commercialisation, soit il n'y a pas de collaborateurs spécialisés, car la fonction n'est pas identifiée. Sur l'ensemble des entreprises de 10 personnes et moins, les deux tiers des entrepreneurs assument seuls la fonction commerciale. Cela nous renvoie à l'opinion générale très répandue dans ces firmes que pour commercialiser les biens il faut 
d'abord connaître la production, le métier. Cette variable d'opinion (question 1) est en effet la plus consensuelle de notre enquête, seules les moins petites entreprises prenant quelques distances avec cette affirmation.

On pourrait penser que ces résultats sont la conséquence d'une méconnaissance, de la part de ces dirigeants, des différents statuts commerciaux et des multiples possibilités qui leur sont offertes d'embaucher ou de contractualiser une personne compétente dans le domaine de la commercialisation. Les résultats de l'enquête infirment cette hypothèse. En effet, les deux tiers des dirigeants des TPE de l'échantillon déclarent avoir une bonne connaissance des statuts commerciaux. En revanche, ils sont près de $80 \%$ à déclarer leur méconnaissance des formations commerciales dispensées par l'université et l'enseignement supérieur en général.

Les données de l'enquête italienne (IMA, 2004) révèlent des résultats similaires. Dans 73 \% des cas, c'est le propriétaire-dirigeant qui a en charge la commercialisation des produits. Si près de $20 \%$ des entreprises interrogées déclarent posséder un service marketing, celui-ci est de fait directement relié au dirigeant. Pour 46,5\% des entreprises interrogées, c'est le dirigeant qui gère ce service. Seules un peu moins de $20 \%$ délèguent cette responsabilité à un cadre.

L'individualisation de la fonction commerciale est corrélée à la taille de l'entreprise: plus l'entreprise grandit, moins elle se montre réticente à embaucher un commercial et la césure entre les très petites entreprises et les petites entreprises est ici forte ${ }^{14}$. Plus de $50 \%$ des firmes de plus de 30 employés ont une ressource commerciale autre que le patron. Il y a donc entre les petites et les moins petites entreprises des différences nettes qui empêchent de les confondre.

Pour la majorité de ces entrepreneurs, la prise en charge de la commercialisation des produits par une personne en particulier présente un inconvénient majeur, celui d'en atténuer le caractère technique et de sousvaloriser le savoir-faire à l'origine du produit. De la même façon, le recours à un commercial atténuerait la relation privilégiée qu'entretient le dirigeant avec ses clients, relation dont la personnalisation est perçue comme l'avantage même de la TPE, sa caractéristique distinctive majeure (Auvolat, Lavigne et Mayère, 1985). Pour les dirigeants des très petites entreprises, la relation avec le client dépend de leur capacité à expliciter le contenu technique du produit et à mettre en relation celui-ci avec les besoins du client. Mais c'est la connaissance intime du produit ou de l'activité qui permet cette rencontre.

14. Cela se vérifie pour les autres fonctions, comme le personnel, par exemple.

Revue internationale P.M.E., vol. 19, nos 3-4, 2006 
Notons que l'augmentation de la taille des entreprises a un effet sur ce rapport particulier au métier. Plus la taille des entreprises augmente, plus elles relativisent l'opinion selon laquelle «dans notre activité et surtout pour discuter avec le client, le plus important c'est de bien connaître le métier. Les techniques et les théories qu'on enseigne à l'université, cela vient bien après et on n'en a pas vraiment besoin ».

Cette relation que le dirigeant souhaite maintenir s'explique aussi par le fait que celui-ci s'estime seul compétent pour retranscrire en actes opérationnels les modifications ou les nouvelles exigences du client. Dans la négociation, le dirigeant perçoit le comment ou les possibilités pour effectuer ces modifications et il est à penser que les informations apportées par des commerciaux ne pourraient relier aussi facilement les exigences des clients et l'évolution rendue nécessaire du savoir-faire. Au-delà des spécificités des dirigeants des TPE, il y a là un véritable problème qui se trouve posé et qui explique aussi, en partie, la grande réticence de ces petites structures à embaucher des jeunes diplômés. Les dirigeants des TPE éprouvent beaucoup de difficultés à faire évoluer leurs compétences et, plus globalement, leur culture d'entreprise. Ces compétences, souvent améliorées et donc façonnées par la relation privilégiée avec un ou quelques clients, se figent rapidement et créent une culture d'entreprise qu'il est difficile de changer. Le lien intime qui se crée entre l'entrepreneur, son savoir-faire et ses clients conduit à une évaluation toute subjective des produits fabriqués, de leur unicité supposée et plus généralement des savoir-faire à l'œuvre dans l'entreprise. On remarque ainsi que sont souvent confondues deux choses: la différenciation dans la manière de concevoir ou fabriquer un produit et le produit lui-même.

Si la première distingue fréquemment les TPE des autres entreprises et notamment de celles de plus grande dimension, il est moins sûr que les produits seraient, eux aussi, toujours différenciés. Comme le font remarquer Bentabet, Michun et Trouvé (1999), une stratégie de spécialisation requiert des capacités matérielles et culturelles, un fort engagement symbolique, notamment dans la publicité, ce à quoi peu de TPE acceptent de (ou peuvent) consentir.

\subsubsection{Le particularisme de la TPE : satisfaire et fidéliser plutôt que conquérir}

Le raisonnement en termes de marché apparaît donc, pour la TPE, comme problématique. Comme nous l'avons dit, la TPE n'envisage pas sa relation à l'environnement autour de la notion d'adaptation à des demandes plus ou moins indifférenciées. Ainsi, Polge (1996) souligne que le marketing est pensé simplement comme une aide visant à faciliter l'insertion d'une capacité dans 
l'environnement, ce qui s'apparente davantage à une démarche commerciale. De même, Marchini (1998) explique que beaucoup d'études tendent à faire entrer dans le concept marketing des réalités qui le plus souvent relèvent de préoccupations commerciales à très court terme. Ce qu'il faut bien saisir, c'est que cette réflexion est menée par rapport à une capacité déjà existante. Il n'y a pas et ne peut y avoir de réflexion afin de faire évoluer cette capacité et de l'adapter aux divers besoins. Dans cette perspective, le besoin d'investigation de nouveaux clients, de nouvelles cibles, est rarement ressenti. Le dirigeant de la TPE raisonne autour d'un savoir-faire déjà constitué et non, a priori, autour de capacités qu'il lui faudrait posséder pour asseoir un avantage concurrentiel quelconque. Comme nous l'avons rappelé, les espaces dans lesquels se meuvent les TPE sont structurés autour de réalités concrètes, identifiables et avec lesquelles elles sont le plus souvent en relation.

De ce point de vue, le raisonnement en termes de menaces et d'opportunités n'apparaît pas adapté. Celles-ci s'évaluent toujours par rapport à des clients avec qui l'on est déjà en contact et non sur la base de scénarii hypothétiques. Le processus d'ajustement, par rapport à la demande, se structure autour de clients et de spécifications explicites.

La notion d'apprentissage par le marché (Day, 1994; Sinkula, 1994) paraît donc, du point de vue de ces entreprises, comme ambiguë; alors que des auteurs (Minguzzi et Passaro, 1995) assimilent trop facilement exigences et caractéristiques des clients avec la sélectivité des marchés. L'approche de «l'écologie des populations», qui sous-tend nombre de ces études (Beatty et Ulrich, 1991; Godener, 2002), finit par occulter l'essentiel en ne cherchant pas à comprendre, en dernier lieu, ce qui fait que ces entreprises ne se préoccupent pas du marché. Lorsque ce problème est abordé, il est à noter qu'il l'est souvent par le biais des profils d'entrepreneurs, ce qui a pour conséquence de créer une coupure toute artificielle entre entreprises qui seraient «dans le coup » et celles qui ne le seraient pas: causalité réductrice et, en dernière instance, peu explicative. Considérer les entreprises peu concernées par les phénomènes de concurrence comme des anomalies ou des entreprises à terme condamnées ne résout pas le problème. De plus, on ne doit pas confondre les spécificités des secteurs dans lesquels les entreprises évoluent et les pressions de la concurrence. L'objectif des dirigeants des TPE est de préserver la régularité en maximisant la satisfaction plutôt que d'élargir l'offre. La satisfaction passe par un service personnalisé et de proximité que les TPE opposent volontiers à l'offre standard des grandes entreprises.

Ainsi, les nouveaux besoins du client sont souvent considérés comme des éléments positifs, des incitations à réfléchir à de nouvelles façons de faire. À ce moment précis de sa relation avec le client, le dirigeant de la 
TPE essaie de les fidéliser en accroissant sa capacité d'accompagnement; ce qui est, somme toute, assez éloigné de la démarche marketing standard, mais assez proche de ce qu'il convient aujourd'hui d'appeler le marketing relationnel (Ferrero, 1992).

Il n'y a pas pour autant rejet en bloc de l'opinion selon laquelle les théories et pratiques commerciales ne sont guère utiles. Les dirigeants des TPE dans leur majorité n'éprouvent pas d'hostilité à l'égard du marketing; ils estiment que celui-ci ne les concerne pas. Une meilleure sensibilité au problème ne changerait que peu de chose à cet état de fait. Ce qui importe pour ces entreprises, c'est de créer une relation durable avec le client, d'être à son écoute; et on le sera d'autant plus que l'on s'estime être en pleine possession de son métier. Si cette maîtrise s'atténue, la perte de la clientèle apparaît comme inévitable. Le rejet constaté à l'égard du marketing et des techniques commerciales en général (Curran, 1988; Watkins et Blackburn, 1986) est directement relié au doute qu'entretiennent les dirigeants quant aux résultats futurs découlant de la mise en place de ces techniques.

Comme le fait remarquer Deret (1999), la qualité de la relation avec le client passe par la chaîne qualité: accueil, écoute, réactivité, contenu technique, aspect relationnel, devis (formalisation, négociation, finalisation), fichier client, suivi de trésorerie. Ce passage a toujours des conséquences sur les compétences de l'entrepreneur et l'oblige souvent à élargir la conception qu'il se fait de son cœur de métier ${ }^{15}$.

\subsection{La gestion de la proximité au cœur de la relation client}

\subsubsection{Des clientèles concentrées et régulières}

Il est clair que l'extrême focalisation des TPE sur leur clientèle et le faible intérêt montré pour tout ce qui touche au marché en général et à la concurrence en particulier révèlent une relation avec l'environnement structurée autour de la proximité (Marchesnay, 1969; Maffesoli, 1988; Torrès, 1998). Comme le soulignent Bentabet, Michun et Trouvé (1999, p. 128), en s'appuyant sur les études statistiques réalisées sur le sujet, «plus la taille de l'entreprise est faible, plus le marché de celle-ci est géographiquement restreint».

L'orientation stratégique des dirigeants des TPE joue ici, aussi, un rôle fondamental. En souhaitant personnaliser sa relation avec l'environnement, le dirigeant de la TPE s'oblige par là même à restreindre son champ

15. Envisagé la plupart du temps autour de la seule compétence technique, du seul savoir-faire.

Revue internationale P.M.E., vol. 19, nºs 3-4, 2006 
d'intervention. De la sorte, ces dirigeants préfèrent des clientèles peu nombreuses et géographiquement proches à un accroissement en volume de celles-ci et à un possible éloignement géographique.

L'étude de Fillis (2002) sur les TPE britanniques révèle des résultats voisins. Par exemple, les entreprises interrogées estiment que leur principale barrière à l'exportation réside dans l'existence d'un marché local qui leur assure un volume d'affaires suffisant. Conquérir de nouvelles clientèles, notamment étrangères, suppose de surcroît des moyens en temps et en ressources dont elles ne disposent pas ou auxquelles elles ne croient pas. Surtout, cette conquête est perçue comme risquée et pas vraiment profitable à long terme. Dans une même perspective, les résultats d'une enquête française sur l'exportation dans les TPE (Fiducial, 2004) révèlent que les raisons principales mises en avant pour justifier la décision de ne pas exporter sont, d'une part, l'absence de nécessité et, d'autre part, le fait que l'activité et le caractère local du marché ne soient pas adaptés à une démarche d'exportation. Ainsi, $51 \%$ de ces entreprises n'éprouvent pas le besoin de conquérir de nouveaux marchés et privilégient les caractéristiques mêmes du marché local, plus adaptées selon elles aux attributs de leurs produits. Une enquête luxembourgeoise (Centre de promotion et de recherche,1992), menée auprès de 959 petits artisans, allait dans le même sens : $42 \%$ des personnes interrogées évoquaient une «demande nationale trop élevée» comme motif de ne pas s'internationaliser.

Les résultats de notre enquête indiquent des taux de concentration des clientèles assez conséquents. Ainsi, près de $40 \%$ du chiffre d'affaires des entreprises de l'échantillon est fourni par trois clients et les trois plus grosses commandes représentent $30 \%$ de ce même chiffre d'affaires. Ces clientèles restreintes présentent l'avantage d'être des clientèles régulières, ces dernières représentant $70 \%$ du chiffre d'affaires des entreprises enquêtées. Ainsi, comme le montre le tableau 6 , les très petites entreprises privilégient nettement les clientèles des particuliers et celles des artisans et PME. Ces choix sont directement reliés à la proximité relationnelle que recherche le dirigeant de la TPE et on se rend compte que les structures de clientèle différencient les très petites entreprises des petites entreprises. Les petites entreprises sont nettement moins réticentes, par exemple, à faire affaire avec la clientèle des grandes entreprises ou des administrations. 
TABLEAU 6

Répartition du chiffre d'affaires selon la clientèle

\begin{tabular}{llllll}
\hline & & & & \\
& 24,72 & 22,95 & 12,70 & 32,11 & 7,52 \\
\hline Total & 16,86 & 29,23 & 6,00 & 44,21 & 3,71 \\
\hline Taille & 17,63 & 21,39 & 14,14 & 38,88 & 7,96 \\
\hline 11 à 6 & 31,04 & 21,29 & 12,43 & 24,19 & 11,06 \\
\hline Plus de 20 & 42,88 & 18,86 & 20,00 & 10,32 & 7,94 \\
\hline
\end{tabular}

Cette proximité est la condition de la réactivité et cette dernière doit donc d'abord s'évaluer par rapport à la capacité de l'entreprise à suivre les exigences de ses clients et non par rapport à un marché en général. Cette recherche de la proximité a sans aucun doute des causes culturelles, mais elle résulte aussi de causes plus objectives, plus rationnelles. Comme le souligne Marchini (1995), l'approfondissement des liens entre les petites entreprises et leurs clients vise à rendre non substituable l'objet de la transaction et par là l'entreprise. Les stratégies d'hyperspécialisation constituent la concrétisation opérationnelle du choix de la gestion de la proximité. La dépendance devient la condition de l'approfondissement. Elle constitue donc pour l'entreprise, à la fois, une force et une faiblesse.

\subsubsection{Une absence d'outils commerciaux}

Il est clair que les outils commerciaux de la TPE découleront de la conception que se font les dirigeants du commercial en général et de la façon dont ils envisagent l'insertion de leur entreprise dans l'environnement. Si les outils proprement mercatiques ne sont pas usités, on peut penser que ceux qui fondent, pourrait-on dire, de façon minimale la démarche commerciale le sont un peu plus. Mais, même ici, les résultats sont peu convaincants. En effet, près des deux tiers des entreprises de l'échantillon ne disposent pas de plaquettes de présentation et près de $80 \%$ d'entre elles n'ont pas réalisé récemment de publipostage. Même si $62 \%$ des TPE déclarent gérer leur 
fichier client sur ordinateur, ce dernier résultat ne doit pas faire illusion, car $70 \%$ de ces entreprises n'ont pas de fichier de prospection $(80 \%$ pour les moins de six salariés).

Une étude menée en 2006 par la fédération des centres de gestion agréés et portant sur la manière dont les TPE répondent aux nouvelles attentes de la clientèle révèle des résultats intéressants. Plus de $55 \%$ des artisans reconnaissent que le degré d'exigence de leurs clients s'est accru de manière significative au cours de ces toutes dernières années. Pour autant, seules $25 \%$ de ces entreprises ont modifié leur stratégie, les autres restant très réticentes à engager des actions de communication ou de fidélisation pour promouvoir leurs atouts. Les résultats de l'enquête italienne (IMA, 2004) révèlent aussi le caractère traditionnel des outils utilisés pour la communication commerciale (catalogues, brochure, foires et expositions). À noter que ces entreprises essaient d'accroître leur notoriété locale par des politiques de commandites, principalement sportives et culturelles. Concernant la connexion au réseau Internet, l'enquête française de Fiducial (2005) montre que $65 \%$ des TPE déclarent être connectées; mais seul un entrepreneur sur quatre déclare disposer d'un site, site qui, dans la moitié des cas, a été conçu en interne.

\section{Conclusion}

Eu égard aux contextes dans lesquels évoluent le plus souvent les très petites entreprises et compte tenu de leur mode particulier d'agrégation à l'environnement, il est indéniable que la relativisation du marketing est plus la résultante d'un choix raisonné et logique qu'un choix proprement culturel.

Les chercheurs doivent s'interroger, par conséquent, sur les a priori qu'ils mobilisent lorsqu'ils qualifient tel ou tel comportement de traditionnel ou d'artisanal. Cette qualification ne s'effectue-t-elle pas en fonction d'un modèle concurrent idéal-type: celui de l'entrepreneur managérial, souvent plus imaginé que réel d'ailleurs (Johannisson, 2003)? Et la théorie de Schumpeter d'un entrepreneur exceptionnel (Ogbor, 2000) n'est-elle jamais loin dans ce cas? Cette projection (Boudon, 1990), souvent inconsciente, peut empêcher l'observateur de trouver les raisons qui inspirent à l'entrepreneur son comportement. Au lieu de cela, on préférera mobiliser des théories plus séduisantes, notamment à base de psychologie, en posant par exemple la question suivante: l'artisan réticent aux technologies de marketing en général n'est-il pas (nécessairement) condamné à disparaître (Mendelsson, 1991)?

L'offre de formation en gestion destinée aux dirigeants des TPE, qu'elle soit de nature consulaire ou autre, repose grandement sur cette vision des choses. Dans tous les cas, l'adaptation au marché devient la seule voie à 
suivre. Le danger d'une telle démarche réside dans le fait que l'on confond deux types de TPE: celles qui ne grandiront pas (les TPE stables) et celles qui grandiront (les TPE émergentes).

On favorise, de la sorte, l'adoption de techniques qui ne correspondent pas aux objectifs des dirigeants. La dissonance, ainsi produite, peut être très dommageable à la compétitivité de l'entreprise parce qu'elle affecte la cohérence même de la démarche stratégique de la TPE (Marchesnay, 1994). A contrario, ce qu'il faudrait pouvoir expliquer, c'est comment des entreprises qui ne développent aucune démarche commerciale font pour survivre, alors même qu'elles évoluent dans des environnements concurrentiels. Dans une perspective plus large, cela devrait conduire à une réflexion sur les conditions de réussite de ces entreprises (O'Donnel et al., 2002). On peut s'interroger si, au fond, on n'aboutit pas avec une telle grille d'analyse à une vision déterministe de la réalité.

De fait, ce travail voulait aussi vérifier si ces entrepreneurs avaient des comportements commerciaux différenciés. Les analyses de variance réalisées ne permettent pas de conclure dans ce sens, que cela concerne la concentration ou la régularité de la clientèle. Toutefois, l'importance accordée à la fonction commerciale varie très sensiblement selon le profil. Dans ce cas, on remarque que les chefs d'entreprise appartenant aux catégories esprit PME et modernes, à la question «fonction commerciale primordiale» obtiennent des résultats partout supérieurs à ceux des chefs d'entreprise appartenant aux catégories passifs, protégés et défensifs. Enfin, sur le plan de l'utilisation des outils (plaquettes, publipostages, fichiers de prospection), on ne note pas de différences significatives.

Ces résultats montrent à l'évidence des appréciations divergentes quant au souhaitable; mais les différences s'estompent sur le possible, c'est-àdire sur leur comportement réel. Cela atténue la pertinence des typologies d'entrepreneurs proposés comme dans notre cas ou chez Woo, Cooper et Dunkelberg (1991). De la même façon, cela démontre que la réticence par rapport au marketing résulte d'une évaluation, somme toute réaliste, des avantages supposés de l'adoption ou non de démarches marketing, eu égard aux contextes dans lesquels évoluent les TPE. De ce point de vue, il est à penser que l'adoption de telles démarches aurait un effet perturbateur certain, car elle remettrait en cause bien des structurants de l'action du dirigeant de la TPE. Ainsi, les dirigeants les plus ouverts préfèrent agir sur des variables qui ne remettent pas en cause leurs choix stratégiques initiaux.

Si l'on note de la part des dirigeants esprit PME et modernes, au vu des résultats de l'enquête, une plus grande capacité à utiliser les services des organismes institutionnels de même qu'une attention plus affirmée pour 
tout ce qui concerne la visibilité externe de leur entreprise, pour autant, ces différences n'entraînent pas des trajectoires comportementales contrastées; et c'est, en dernier lieu, ce qui nous paraît important de souligner.

L'ouverture sur l'extérieur ne signifie pas ouverture sur le marché ou un comportement marketing accentué. L'ouverture se situe plutôt dans la capacité de l'entreprise à mobiliser des ressources liées à l'information pour répondre aux nouvelles exigences des clients.

Dès lors, il devient nécessaire lorsqu'on examine les profils des dirigeants de TPE d'établir une distinction nette entre ce qui concerne, d'une part, le rapport à l'entreprise et, d'autre part, le rapport à l'environnement. C'est seulement en fonction du second que s'opère la différenciation des comportements. Mais globalement, le rapport à l'entreprise reste le même, la TPE «marketing» ne constituant pas, tant s'en faut, une PME en devenir.

D'un point de vue plus pratique, les stratégies de satisfaction qui réussiront seront aussi celles qui s'appuieront sur une connaissance intime du client, pas simplement perçue. De la sorte, une sensibilisation plus affirmée des besoins du client et de la nécessité de s'y adapter feront évoluer la nature du cœur de métier de l'entrepreneur et de la perception qu'il pourra en avoir. Dans cette perspective, un marketing entrepreneurial (Carson et al., 1995) a toute sa place dans la TPE. Ce que le dirigeant de la TPE doit approfondir, ce sont les paramètres sur lesquels il fonde la connaissance de ses clients. Sans remettre en cause les principaux constituants, il faut élargir la vision de l'entrepreneur et le sensibiliser aux techniques qui lui permettent d'approfondir cette connaissance. De ce point de vue, le recours à un commercial n'est pas forcément la solution. Le véritable défi pour les dirigeants des TPE réside dans leur capacité à envisager la satisfaction du client autrement que sur les seuls aspects techniques.

Le marketing ne peut être soluble dans la TPE qu'à la condition d'accompagner pour parfaire des démarches plutôt que pour les modifier. Le marketing de la TPE devra donc s'efforcer d'améliorer la capacité d'accompagnement des TPE relativement à leurs clientèles plutôt que d'aider à la conquête de nouvelles. Un changement de perspective qui peut marquer le début d'une rencontre fructueuse.

\section{Bibliographie}

Auvolat, M., J.C. Lavigne et A. Mayère (1985), L'artisanat en France: réflexion générale et prospective, Paris, La Documentation française.

Amendola, M. et J.L. GafFard (1988), La dynamique économique de l'innovation, Paris, Economica. 
BALDwin, J.R. et P.K. GoReCKI (1991), «Firm entry and exist in the Canadian manufacturing sector», Canadian Journal of Economics, vol. 24, n 3, p. 300-323.

BEATTY, R.W. et D.O. UlRich (1991), «Re-energizing the nature of organizations », Organizational Dynamics, vol. 20, $\mathrm{n}^{\circ}$ 1, p. 16-31.

Bentabet, E., S. Michun et P. Trouvé (1999), «Gestion des hommes et formation dans les très petites entreprises », Étude n ${ }^{\circ} 72$, CÉREQ.

Boudon, R. (1990), L'art de se persuader, Paris, Fayard.

CAndau, P. (1981), «Pour une taxonomie de l'hypofirme», Revue d'économie industrielle, $\mathrm{n}^{\circ} 16, \mathrm{p} .16-33$

Carson, D., S. Cromie, P. McGovan et J. Hill (1995), Marketing and Entrepreneurship in SMEs. An Innovative Approach, Londres, Prentice-Hall.

Carson, D. et A. Gilmore (2001), «The theory/practice divide», European Journal of Marketing, vol. 35, $\mathrm{n}^{\text {os }}$ 5-6, p. 521-523.

CENTRE de PROMOTION ET DE RECHERCHE (1992), «Enquête sur l'activité d'exportation et la participation des entreprises du secteur de l'artisanat aux foires nationales et internationales», Chambre des métiers du Grand Duché du Luxembourg, Luxembourg.

Chicha, J. et P.-A. Julien (1980), «Les stratégies des PME et leur adaptation au changement», Cahiers de recherche du GREPME, n 80-05, Université du Québec à Trois-Rivières.

CozzI, G. (1986), «Il marketing nelle piccole e medie imprese», Economia Delle Piccole e Medie Imprese, Atti Seminaro AIDEA club, Bologne.

Coviello, N.E., R.J. Brodie et H.J. Munro (2000), «An investigation of marketing practice by firm size», Journal of Business Venturing, vol. 15, n 5 , p. 523-545.

Curran, J. (1988), «Training and research strategies for small firms», Journal of General Management, vol. 13, n 3, p. 24-37.

DAY, G. (1994), «Continuous learning about markets», California Management Review, vol. 36, no 4. p. 9-31.

DAvid, M. (1998), «Brève histoire de l'artisanat», Cahier de l'Institut supérieur des métiers, Paris.

Davidsson, P. (1989), «Entrepreneurship and after? A study of growth willingness in small firms », Journal of Business Venturing, vol. 4, n 3, p. 211-226.

DenNis, W.J. (1987), «Small retailer forecast of next quarter sales: how accurate?» dans G.E. Hills (dir.), Research at the Marketing Entrepreneurship Interface, Chicago, University of Illnois.

Deret, E. (1999), «Produire pour un marché, quelles compétences?», dans Artisans et stratégie commerciale, Cahier de l'Institut supérieur des métiers, Paris.

ObSERVATOIRE DE LA PETITE ENTREPRISE DE LA FÉDÉRATION DES CENTRES DE GESTION AGRÉÉs (2006), Les clients des TPE en veulent toujours plus!, n 19.

Ferrero, G. (1992), Il marketing relazionale. L'approchio delle scuole nordiche, Trieste, Lint.

Revue internationale P.M.E., vol. 19, nºs 3-4, 2006

(C) 2006 - Presses de l'Université du Québec

Édifice Le Delta I, 2875, boul. Laurier, bureau 450, Québec, Québec G1V 2M2 • Tél.: (418) 657-4399 - www.puq.ca 
Fiducial (2004), Baromètre des PME, février.

FiLION, L.J. (1996), «Un système marketing pour les travailleurs autonomes: développer ses réseaux pairs et complémentaires", Cahier de recherche HECMontréal, no 96-11-03.

FiLLIs, I. (2002), «Barriers to internationalisation: an investigation of the craft microenterprise », European Journal of Marketing, vol. 36, n ${ }^{\text {os }} 7-8$, p. 9120927.

GibB, A.A. et M. Sсотт (1986), «Understanding small firms growth», dans M. Scott, A. Gibb, J. Lewis et T. Faulkner (dir.), Small Firms: Growth and Development, Aldershot, Angleterre, Gower Publishing, p. 81-104.

Gilmore, A., D. Carson et K. Grant (2001), «SME marketing in practice », Marketing Intelligence and Planning, vol. 19, $\mathrm{n}^{\circ}$ 1, p. 6-11.

GODENER, A. (2002), «PME en croissance: peut-on prévoir les seuils organisationnels? », Revue international PME, vol. 15, $\mathrm{n}^{\circ}$ 1, p. 39-64.

Herrmann, J.L. (1998), «Le marketing en PME manufacturières, les pratiques de quelques entreprises lorraines lors du développement du produit», Actes du IV Congrès francophone sur la PME, 22-24 octobre, Nancy, Metz.

Hills, G.E. (dir.) (1987), «Marketing and entrepreneurship research issues: scholarly justification », Research Symposium on the Marketing, Entrepreneurship Interface, Chicago, comptes rendus, p. 3-15.

IstiTUTO MARKETING AGROALIMANTARE - IMA (2004), «Il marketing nelle PMI agroalimentari piemontese: un'indagine sui comportamenti e sulle attese, per attuare solizioni vincenti», Turin.

JoHANNISSON, B. (2003), «La modernisation des districts industriels : rajeunissement ou colonisation managériale? », Revue internationale PME, vol. 16, nº 1, p. 11-42.

Julien, P.-A. (1995), «New technologies and technological information in small businesses », Journal of Business Venturing, vol. 10, nº 6, p. 459-476.

Julien, P.-A., R. LAchance et M. Morin (2005), «Réseaux personels, d'affaires et informationnels : signaux forts et faibles et innovation », Revue de management technologique, numéro thématique sur les réseaux personnels (à paraître).

Julien, P.-A., P. Mustar et M.F. Estimé (2001), «Les PME à forte croissance: une comparaison internationale ", éditorial du numéro thématique sur les PME à forte croissance, Revue internationale PME, vol. 14, nos 3-4, p. 7-16.

KAminski, P. (1995), «Comment aider la TPE de technologie?», Cahier de recherche $I R G, \mathrm{n}^{\circ} 95.08$, Université Paris XII.

KAminski, P. et J.-C. PacitTo (2000), «Formation et innovation: le cas des très petites entreprises industrielles françaises», Congrès international francophone sur les PME, octobre, Lille.

KinSEY, J. (1987), «Marketing and the small manufacturing firms in Scotland: findings of a pilot study», Journal of Small Business Management, vol. 25, nº 2, p. 18-25.

Kotler, P. (1999). Le marketing selon Kotler, Paris, Village Mondial.

Revue internationale P.M.E., vol. 19, nos 3-4, 2006 
Le Cornu, M.R., R.G.P. McMahon, D.V. Forsaith et M.J. Stanger (1996), «The small enterprise financial objective function», Journal of Small Business Management, vol. 34, $\mathrm{n}^{\circ}$ 3, p. 1-14.

Letowski, A. (1987), Les systèmes socio-culturels de l'artisanat face aux mutations, Thèse de science des organisations, Université Paris Dauphine.

MAFFesoli, M. (1988), Le temps des tribus. Le déclin de l'individualisme dans les sociétés de masse, Paris, Méridiens Klincksieck.

Marchesnay, M. (1969), Analyse dynamique et théorie de la firme-Contribution à une théorie générale de l'entreprise, Thèse de doctorat en sciences économiques, Paris, 10 mai.

Marchesnay, M.(1988), «La mercatique de la petite entreprise », Revue internationale PME, vol. 1, nos 3-4, p. 259-276.

Marchesnay, M. (1994), «Le management stratégique», dans P.-A. Julien (dir.), Les PME. Bilan et perspectives, Paris, Economica, p. 133-162.

MARChINI, I. (1995), Il governo delle piccola impresa, vol. 1, Le basi delle conoscenze, Gênes, INS-EDIT, 150 p.

MARChini, I. (1997), «Il difficile rapporto tra piccola impresa e marketing; lo stato delle conoscenze », Piccola Impresa, 1-1997, p. 3-12.

MARChINI, I. (1998), Il governo della piccola impresa, Gênes, INS-EDIT.

Mendelsson, J. (1991), «Small firm and the marketing mission», Accountancy, vol. 107, $\mathrm{n}^{\circ}$ 1167, p. 96-97.

Minguzzi, A. et R. PAssaro, (1995), «L'apprentissage comme facteur d'innovation des entreprises. Enquête et exploration dans les petites entreprises italiennes », Actes du congrès «Innovation et organisation des PME», Paris.

NARVER, J.C. et S.F. SLATER (1990), «The effect of a market orientation on business profitability», Journal of Marketing, vol. 54 n 4, p. 20-35.

Nexima (2005), Du marketing dans ma PME, Rennes.

Organisation dE COOPÉRATION ET DE DÉVELOPPEMENT ÉCONOMiQues-OCDE (2002), Perspectives de l'OCDE sur les PME, Paris, OCDE.

O'Donnell A., A. Gilmore, D. CARson et D. Cummins (2002), « Competitive advantage in small and medium-sized enterprises », Journal of Strategic Marketing, vol. 10, $\mathrm{n}^{\circ} 3$, p. 205-223.

Ogbor, J.O. (2000), «Mythicizing and reification in entrepreneurial discourse: Ideology-critique of entrepreneurial studies », Journal of Management Studies, vol. $37, \mathrm{n}^{\circ} 5$, p. 605-635.

Ohmae, K. (1983), «The strategic triangle and business unit strategy», The McKinsey Quarterly, p. 9-24.

Pacitto, J.-C., P.-A. Julien et O. Meier (2002), «Les TPE sont-elles spécifiques?», Piccola Impresa/Small Business, n² 234, p. 35-61.

PacitTo, J.-C. et F. Tordjman (2000), «Très petite entreprise et marketing: les causes d'un malentendu », Revue internationale PME, vol. 13, n ${ }^{\text {os }} 3-4$, p. 37-62.

Revue internationale P.M.E., vol. 19, $\mathrm{n}^{\text {os }} 3-4,2006$

(C) 2006 - Presses de l'Université du Québec

Édifice Le Delta I, 2875, boul. Laurier, bureau 450, Québec, Québec G1V 2M2 • Tél.: (418) 657-4399 - www.puq.ca

Tiré de: Revue internationale P.M.E., vol. 19, nos 3-4, sous la direction de Louis Raymond - PME1903N Tous droits de reproduction, de traduction et d'adaptation réservés 
Perreault, J.D. (2005), «Le marketing de la PME», dans P.-A. Julien (dir), Les PME. Bilan et perspectives, Québec, Presses Inter Universitaires, p. 163-188.

Peterson, R.T. (1989), «Small business adoption of the marketing concept vs other business strategies», Journal of Small Business Management, vol. 27, ${ }^{\circ} 1$, p. 38-46.

Polge, M. (1996), L'avantage concurrentiel en petite entreprise, Thèse de doctorat, Université de Montpellier, janvier.

Romano, C. et J. RatnAtunga (1995), «The role of marketing. Its impact on small enterprise research », European Journal of Marketing, vol. 29, n 7, p. 9-30.

Rosa, P. et R. Hale (1990), «Hale the craft ideology as a barrier», Piccola Impresal Small Business, ${ }^{\circ}$ 1, p. 27-45.

SCASE, R. et R. Goffee (1980), The Real World of the Smaller Business Owner, Londres, Croom Helm.

Siméoni, M. (1998), «Le rôle du savoir-faire dans la firme artisanale», Actes du $I V^{e}$ Congrès francophone sur la PME, 22 au 24 octobre, Nancy, Metz.

SinKula, J.M. (1994), «Market information processing and organizational theory», Journal of Marketing, vol. 58, $\mathrm{n}^{\circ}$ 1, p. 35-45.

Smeltzer, L., G.L. Fann et V.K. Nicolaisen (1988), «Environmental scanning practices in small business », Journal of Small Business Management, vol. 26, $\mathrm{n}^{\circ} 1, \mathrm{p} .55-64$.

Stanworth, J. et J. Curran (1973), Management Motivation in Smaller Business, Farnborough, Gower Press.

Strauss, A.L. et J. Corbin (1998), Basics of Qualitative Research. Techniques and Procedures for Developing Grounded Theory, $2^{\mathrm{e}}$ édition, Thousand Oaks, Sage.

Théron, J.L. (2001), «De l'approche marché dans les entreprises artisanales», Document ISM.

TORRÈs, O. (1998), PME: de nouvelles approches, Paris, Economica.

WALter, F. et A. BRown (2004), «What success fartors are important to small business owners? », International Small Business Journal, vol. 22, n 6 , p. 577-594.

Watkins, T. et B. BlackBurn (1986), «The role of marketing in the small firm: evidence from a regional survey», Marketing Intelligence and Planning, vol. 4, $\mathrm{n}^{\mathrm{o}} 4$, p. 26-38.

Werman, M, M.S. Span et M. AdAms (1989), «The interface of entrepreneurship and marketing: concept and research perspective », dans G.H. Hills, R.W. La Forge et B.J. Baker (dir.), Research at the Marketing/Entrepreneurship Interface, Chicago, University of Illinois.

Woo, C.Y., A.C. Cooper et W.C. Dunkelberg (1991), «The development and interpretation of entrepreneurial typologies », Journal of Business Venturing, vol. 6 , $\mathrm{n}^{\circ}$ 2, p. 93-111.

Zdatny, S. (1998), Les artisans en France. Une entreprise humaine, Paris, Belin.

Revue internationale P.M.E., vol. 19, nºs 3-4, 2006 